\title{
FDA Unique Device Identifier
}

National Cancer Institute

\section{Source}

National Cancer Institute. FDA Unique Device Identifier. NCI Thesaurus. Code C112279.

A unique numeric or alphanumeric code that contains the device identifier and a

production identifier such as the lot or batch number, the serial number and/or expiration date. 